\title{
Experimental study of volume speckle in four-wave mixing arrangement
}

\author{
Angel Salazar $^{\mathrm{a}}$, Myrian Tebaldi ${ }^{\mathrm{b}, *}$, Néstor Bolognini ${ }^{\mathrm{b}, 1}$ \\ a Universidad Pontificia Bolivariana, Medellin, Colombia

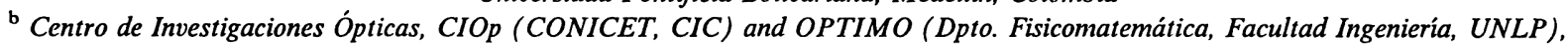 \\ P.O. Box 124, La Plata (1900), Argentina
}

Received 20 January 2003; received in revised form 2 May 2003; accepted 8 May 2003

\begin{abstract}
We study a high frequency modulated speckle pattern in a four-wave mixing arrangement. The three-dimensional nature of the speckles is considered in the phase conjugate reflectivity evaluation which implies to analyze its dependence on the average speckle size. Also, the reflectivity is analyzed in terms of the external applied field, the probe beam ratio, and the pupil aperture diameter of the imaging system that generates the subjective speckle beam.
\end{abstract}

(c) 2003 Elsevier Science B.V. All rights reserved.

PACS: 42.30.Ms; 42.30.Va; $42.40 . \mathrm{Ht}$

Keywords: Speckle; Photorefractive BSO crystal

\section{Introduction}

The photorefractive crystals of the sillenite family have a high photosensitivity and high carrier mobility which permit achievements of fast response time that makes them adequate for real time holography, optical phase conjugation, amplification of weak light signals, and image processing [1-3]. For example, the phase conjugation could be applied to the transmission of optical

\footnotetext{
"Corresponding author. Tel.: +54-221-4840280; fax: +54 221-4712771.

E-mail address: myrianc@ciop.unlp.edu.ar (M. Tebaldi).

${ }^{1}$ Also Facultad de Ciencias Exactas, UNLP, Argentina.
}

waves through scattering media such as the atmosphere.

The first experience of phase conjugation in a photorefractive material was done by Huignard et al. [4]. Applications of phase conjugation to nondestructive testing, image processing, and imaging through phase disturbing media have been already demonstrated [5-9]. In [10] a real time double exposure holographic interferometric method of testing phase object by using degenerate four-wave mixing DFWM with photorefractive $\mathrm{Bi}_{12} \mathrm{GeO}_{20}$ (BGO) crystals has been reported.

As it is well known, when a diffusing surface is illuminated by coherent light, speckles appear in front of the surface. The speckle patterns have important applications in the field of metrology 
and image processing [11,12]. Displacements, tilts, and deformations of the input diffuser produce displacements and structural changes in the speckle patterns, which can be analyzed on the basis of the double-exposure imaged speckles, before and after deformation. In previous papers, the use of a thick photorefractive crystal as a speckle recording medium was proposed [13-16]. In [1719], the storage in a sillenite crystal of low frequency modulated speckle patterns is analyzed. In this case, the image of a coherently illuminated diffuser is formed onto the crystal by an optical system whose pupil consists of two or more identical apertures. In addition, it has been investigated that the features of a multiple exposure specklegram are obtained by using different multiple aperture arrangements for recording [19]. Standard couple wave theory proved to be an adequate theoretical frame to interpret the different experiments mentioned above, which take advantage of the three-dimensional nature of the speckle patterns.

These previous results encourage us to introduce a new experiment where a speckled input signal is high frequency fringe modulated in a four-wave mixing arrangement. The precise knowledge of the speckle pattern recording parameters in BSO constitutes an important feature for image speckle applications. We present an analysis in which the volume speckle nature is accounted for. Three-dimensional speckles must be considered in the phase conjugate reflectivity evaluation which implies to analyze its dependence on the average speckle size. The speckle size can be easily adjusted by modifying the imaging system parameters, in our case, the pupil aperture diameter. We present in this paper an analysis of the reflectivity in terms of the external applied field, the probe beam ratio, and the pupil aperture diameter of the imaging system that generates the subjective speckle beam. Our experimental results are interpreted on the basis of the theoretical calculation proposed in [20].

It should be pointed out that the precise knowledge of the reflectivity behavior leads to the adequate selection of the experimental parameters in speckle applications concerning optical signal processing and metrology.

\section{Experimental arrangement and theoretical model}

Four wave mixing is a convenient method for the generation of phase conjugated waves. When a signal beam is incident into a nonlinear medium, a fourth beam is generated provided that a pair of counterpropagating laser beams exist in the medium. Referring to the experimental arrangement of Fig. 1, we consider the interaction of four beams in the photorefractive medium which can be schematized as is shown in Fig. 2.

In Fig. 1, the wavefront denoted as $A_{4}$ is the signal beam which corresponds to the image of the diffuser $R$ formed onto the photorefractive BSO crystal by lens $L_{1}$. A pupil aperture of diameter $D$ is located immediately in front of lens $L_{1}$. The parameters $Z_{0}$ and $Z_{\mathrm{C}}$ denote the distance from the diffuser to the lens and from the lens to the crystal, respectively. The BSO crystal is cut normal to the $\langle\overline{1} 10\rangle$ crystallographic direction (transverse electro-optic configuration). Moreover, the directions $\langle\overline{1} 10\rangle,\langle 001\rangle$, and $\langle 110\rangle$ coincide with the $X Y Z$ axes, respectively. Let us denote $A_{i}(i \equiv 1, \ldots, 4)$, the amplitudes of the interfering beams. In the crystal, the speckle beam $A_{4}$ interferes with the pump beam $A_{1}$ so that the three-dimensional image speckle grains are fringe modulated. This intensity distribution generates a space charge field in the crystal due to a charge carrier redistribution.

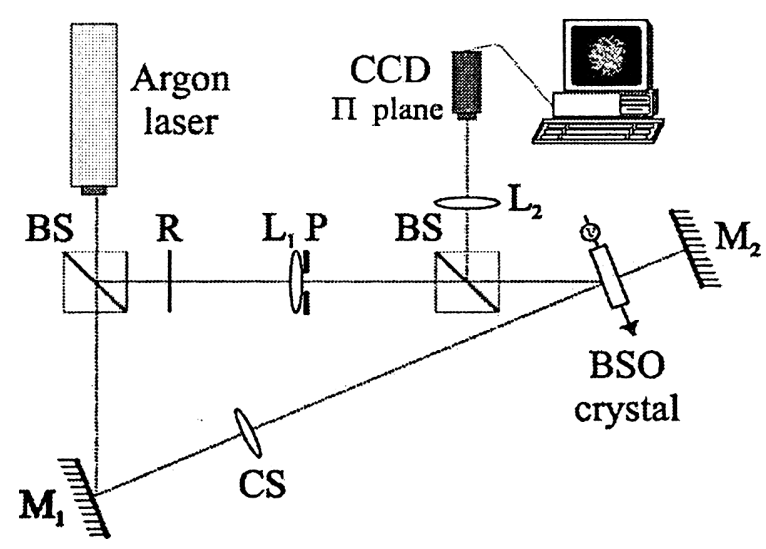

Fig. 1. Experimental set-up: $R$, diffuser; $L_{1}$ and $L_{2}$, lenses; CS, collimation system; $M_{1}$ and $M_{2}$, mirrors; $\Pi$, detection plane. The pupil aperture $P$ of diameter $D$ discussed along the text is attached to the lens $L_{1}$. 


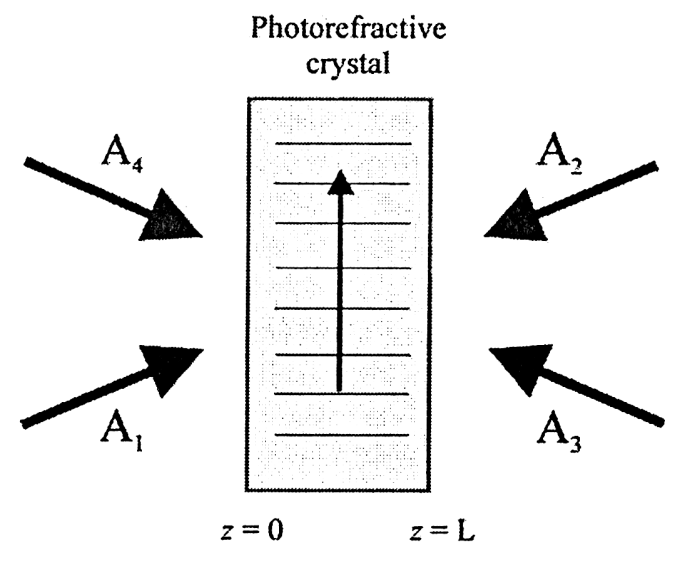

Fig. 2. Transmission grating scheme in a four-wave mixing arrangement.

As a consequence of the linear electro-optical effect that the crystal exhibits, the resulting space charge field produces a refractive index perturbation which replicates and stores the speckle intensity distribution. The transmitted part of beam $A_{1}$ is retroreflected by mirror $M_{2}$ as beam $A_{2}$, so that in the resulting wave mixing inside the crystal, the beam $A_{3}$ is generated and is proportional to the conjugate of the image speckle bearing beam $A_{4}$. Other index gratings are produced in the wave mixing phenomenon.

The predominance of one grating is common in practical situations due to the space charge field dependence on the grating period, grating orientation, and coherence between the beams. This condition is valid in our case.

An exact solution for the coupling equations is given in [20]. The solution considers only transmission gratings, which implies that the grating associated to $A_{1} A_{4}^{*}+A_{2}^{*} A_{3}$ only couples strongly the beams. This solution considers that the pump beam loses energy in the mixing procedure. By taking into account the conservation laws, it is possible to uncouple the equations and find the amplitudes $A_{i}(z)(i=1, \ldots, 4)$ throughout the nonlinear medium, which extends between $z=0$ and $z=L$. After calculation [20], it is possible to obtain the phase conjugate reflectivity $R$

$R=\left|\frac{A_{3}(0)}{A_{4}^{*}(0)}\right|^{2}=\left|\frac{-2 C \tanh (\mu L)}{\Delta \tanh (\mu L)+\left(\Delta^{2}+4|C|^{2}\right)^{1 / 2}}\right|^{2}$, where

$\mu=\frac{\gamma\left(\Delta^{2}+4|C|^{2}\right)^{1 / 2}}{2 \sum_{i=1, \ldots, 4} I_{i}}$

$\Delta=I_{2}+I_{3}-I_{1}-I_{4} \quad\left(I_{i}=A_{i} A_{i}^{*}, i=1, \ldots, 4\right)$,

$C=A_{1} A_{2}+A_{3} A_{4}$,

and the coupling constant $\gamma$ is

$\gamma=\frac{\mathrm{i} \pi n_{1} \exp (-\mathrm{i} \varphi)}{\lambda \cos \theta}$,

where $\lambda$ is the wavelength of the beams, $\theta$ is the half angle between the incident beams,

$n_{1}=\frac{r_{41} n_{0}^{3} E_{q}}{2}\left[\frac{E_{0}^{2}+E_{D}^{2}}{E_{0}^{2}+\left(E_{D}+E_{q}\right)^{2}}\right]^{1 / 2}$ and
$\tan \varphi=\frac{E_{D}\left(E_{D}+E_{q}\right)+E_{0}^{2}}{E_{0} E_{q}}$

[21], $r_{41}$ is the electro-optic coefficient, $n_{0}$ is the refractive index at the incident wavelength, $E_{D}=(k T / q)(2 \pi / \Lambda)$ is the diffusion field $(T$, temperature; $k$, Boltzmann constant; $q$, electron charge; $\Lambda \equiv \lambda / 2 \sin \theta$, grating period), $E_{q}=\left(N_{\mathrm{A}} q /\right.$ $\left.\varepsilon_{0} \varepsilon_{S}\right)(\Lambda / 2 \pi)$ is the maximum space charge field $\left(N_{\mathrm{A}}\right.$, acceptor density; $\varepsilon_{0}$, vaccum permittivity; $\varepsilon_{\mathrm{S}}$, relative permittivity), and $E_{0}$, the external applied field.

By considering the boundary conditions, the parameter $|C|^{2}$ in Eq. (1) is obtained as a solution of equation

$$
\begin{aligned}
f\left(|C|^{2}\right)= & \left(|C|^{2}-I_{1}(0) I_{2}(L)\right) \mid \Delta \tanh (\mu L) \\
& +\left.\left(\Delta^{2}+4|C|^{2}\right)^{1 / 2}\right|^{2} \\
& +4|C|^{2}|\tanh (\mu L)|^{2} I_{4}(0) I_{2}(L) \\
& +2|C|^{2} I_{4}(0)\left(\Delta^{2}+4|C|^{2}\right)^{1 / 2} \\
& \times\left(\tanh (\mu L)+\tanh ^{*}(\mu L)\right)=0 .
\end{aligned}
$$

It is convenient to define the probe beam ratio as

$q=\frac{I_{4}(0)}{I_{1}(0)+I_{2}(L)}$

and the pump beam ratio as

$r=\frac{I_{2}(L)}{I_{1}(0)}$. 


\section{Analysis of the experimental results}

In our experimental arrangement the dimension of the BSO crystal is: $10 \mathrm{~mm} \times 10 \mathrm{~mm} \times 10 \mathrm{~mm}$. To perform the theoretical calculation, the parameter values are: $N_{\mathrm{A}} \equiv 0.95 \times 10^{22} \mathrm{~m}^{-3}$ [2,22], $\varepsilon_{\mathrm{S}} \equiv 56, n_{0} \equiv 2.63, r_{41} \equiv 4 \times 10^{-12} \mathrm{~m} / \mathrm{V}$. An Argon laser is employed $(\lambda \equiv 514 \mathrm{~nm})$. The half incident angle is $27.63^{\circ}$. Then, the average grating period is $0.56 \mu \mathrm{m}$ at $\lambda \equiv 514 \mathrm{~nm}$.

Let us consider a diffuser that is rough in the scale of the optical wavelength. Under illumination by monochromatic light, the wave transmitted consists of contributions from many different scattering points. The image formed at a given point in the observation plane consists of a superposition of a multitude of amplitude spread functions, each arising from a different scattering point on the surface of the object. As a consequence of the surface roughness the various spread functions add with random phases resulting in a highly complex pattern of interference called speckle. Therefore, the image field is characterized by a randomly varying intensity and phase. The statistical properties of the speckle intensity and phase have been largely studied [11,12].

It should be taken into account that the model detailed in the previous section describes a situation where the beams $A_{3}$ and $A_{4}$ are uniform. In our case, this situation does not hold. It should be emphasized that the recorder index gratings are random in phase according to the incident speckle distribution. Each of these gratings interacts with the counterpropagating pump beam thus producing a local phase conjugated beam inside each speckle grain with random phase that "remembers" the original local speckle phase. Therefore, the reconstructed beam $A_{3}$ is a phase conjugate beam which is a speckle pattern itself governed by the same statistics as the input beam. To consider the statistical behavior, an average amplitude and intensity will be employed in the phase conjugate reflectivity calculations. That is the validity of considering average values and comparing them with the experimental results will be analyzed.

Another feature of the speckle pattern to be taken into account is its volume nature. The average diameter and length of the speckle grains are $\left\langle S_{X}\right\rangle \approx \lambda \cdot\left(Z_{C} / D\right)$ and $\left\langle S_{Z}\right\rangle \approx \lambda \cdot\left(Z_{C} / D\right)^{2}$, respectively ( $D$ is the lens aperture diameter) [11]. Then, the speckle dimensions decrease in proportion as the aperture diameter increases. Owing that $Z_{C}$ remains fixed along the experiments, the parameter $D$ governs the speckle size.

The phase conjugate reflectivity depends on the parameter $L$ (see Eq. (1)). Usually $L$ coincides with the crystal depth. Note that in the proposed speckle arrangement, the wave mixing is produced inside each speckle grain. Then, in reflectivity theoretical calculation the crystal depth must be replaced by the speckle depth if the speckle grain is shorter than the crystal depth.

In accordance with the experimental arrangement values, let us fix the imaging distance $Z_{C}=300 \mathrm{~mm}$. For instance, for a pupil aperture $D \equiv 2 \mathrm{~mm}$, the average speckle depth is $\left\langle S_{Z}\right\rangle \approx 11$ $\mathrm{mm}$, whereas for $D=20 \mathrm{~mm},\left\langle S_{Z}\right\rangle \approx 0.11 \mathrm{~mm}$. Therefore, according to the pupil aperture diameter, it should be evaluated which is the correct value of the parameter $L$ in the theoretical model to be utilized, in particular see Eq. (1). For example, let us consider a 10 -mm crystal thickness, then for $D=8 \mathrm{~mm}$ which implies $\left\langle S_{Z}\right\rangle \approx 0.7 \mathrm{~mm}$, the replacement of the parameter $L$ by the crystal thickness brings an incorrect evaluation of the phase conjugate reflectivity because the speckle length is shorter than the crystal depth. Then, the parameter $L$ in Eq. (1) must be written as

$L= \begin{cases}\left\langle S_{Z}\right\rangle & \text { if }\left\langle S_{Z}\right\rangle<\text { crystal thickness } \\ \text { crystal thickness } & \text { otherwise. }\end{cases}$

The total phase conjugate reflectivity is proportional to the phase conjugate reflectivity associated to an individual volume speckle grating. That is to evaluate the total reflectivity, the reflectivity belonging to a single speckle grain must be multiplied by an adequate scale factor related to the density of speckle grains.

Another point to be considered in the experimental data analysis is related with the speckle orientation. It should be noted that the on-axis speckle grains ( $Z$-axis) are oriented along the crystal depth direction, whereas the inclination of 
the off-axis speckle grains with respect to the $Z$ axis changes. Then, the interference between the speckle signal beam and the pump beams generates an ensemble of index gratings which has associated a dispersion $\Delta \vec{K}$ in wave vector space $\vec{K}$ related to $A_{1} A_{4}^{*}+A_{2}^{*} A_{3}$. Note that the dispersion in $\vec{K}$ values depends on the pupil aperture diameter of the imaging lens. Therefore, an ensemble of gratings with different orientations is produced. This behavior is confirmed by observing Fig. 3 where the phase conjugate image beam and the corresponding intensity profile by using pupil aperture diameter $D=2$ and $5 \mathrm{~mm}$ are shown. In both cases, the crystal thickness is $10 \mathrm{~mm}$ and the pump beam ratio is fixed at $r \equiv 1.138 \times 10^{-1}$. Note that on an average, the reflectivity profile belonging to $D=5 \mathrm{~mm}$ is less intense and broadened in comparison with the profile belonging to $D=2 \mathrm{~mm}$. This broadening confirms that the diffraction spots corresponding to larger pupil aperture diameters have a higher dispersion in $\vec{K}$ vector space.

The experimental data of this section are obtained from the phase conjugate beam. This beam is itself a speckle distribution. Then, to obtain the experimental data, an average intensity profile is considered.

The experimental probe and pump ratio values are obtained by measuring the average intensity of the beams at the crystal.

On this basis, in the following, different experimental situations are evaluated by considering the dependence of the phase conjugate reflectivity on the parameters that govern the image speckle grain size, the beam intensities, and the external applied field.

\subsection{Reflectivity vs. probe ratio}

In this case, the pump beam intensities remain fixed as well as the lens pupil aperture diameter, whereas the speckle beam average intensity is modified. By fixing the lens pupil aperture diameter, the speckle size remains unchanged. Let us remember that the probe beam ratio $q$ is defined as the signal (speckle) beam average intensity divided by the sum of both average pump beams, see
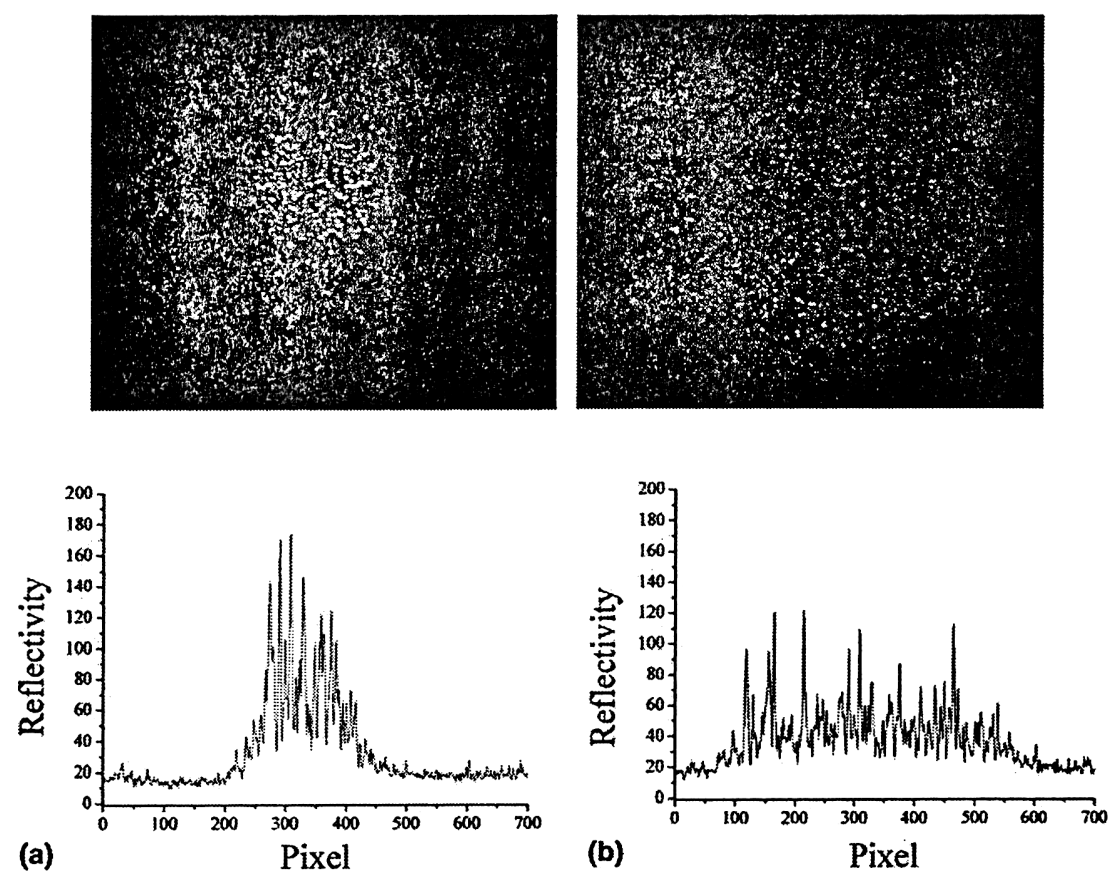

Fig. 3. Phase conjugate reflectivity image beam and their corresponding intensity profile: (a) $D=2 \mathrm{~mm}$, (b) $D=5 \mathrm{~mm}(L=10 \mathrm{~mm}$, $\left.r=1.32 \times 10^{-1}\right)$. 
Eq. (7). When the average amplitude of the speckle beam is modified, the grating amplitudes $A_{1} A_{4}^{*}+A_{2}^{*} A_{3}$ change and therefore the modulation of the photorefractive gratings inside the speckle grains change as well.

In Fig. 4, the theoretical and experimental reflectivity values in terms of the probe beam ratio for a 2-mm pupil aperture diameter are shown. In all cases, the crystal thickness is $10 \mathrm{~mm}$. The average pump beams remain unchanged in all the experiments and the pump beam ratio defined as the ratio between the pump beams is fixed at $r=1.428 \times 10^{-1}$. To determine the experimental probe beam ratio values, the intensities $I_{4}(0), I_{1}(0)$, and $I_{2}(L)$ at the crystal faces are measured. The speckle beam average intensity $I_{4}(0)$ is modified in our experiments producing a change in the speckle grain modulation. By increasing the speckle beam average intensity $I_{4}(0)$, both the probe beam ratio and the speckle grain modulation will increase and therefore the conjugate beam intensity $I_{3}(0)$ increases as well. Experimental results of the phase conjugation reflectivity as a function of the probe beam ratio are shown in Fig. 4. Let us remember that the phase conjugation reflectivity $R$ is obtained as the ratio between the average intensities $I_{3}(0)$ and $I_{4}(0)$. It is observed that the phase conjugate reflectivity increases as the probe beam ratio increases. Also, in Fig. 4 a theoretical curve obtained by using Eq. (1) is shown. To perform the theoretical calculations, the parameters values in-

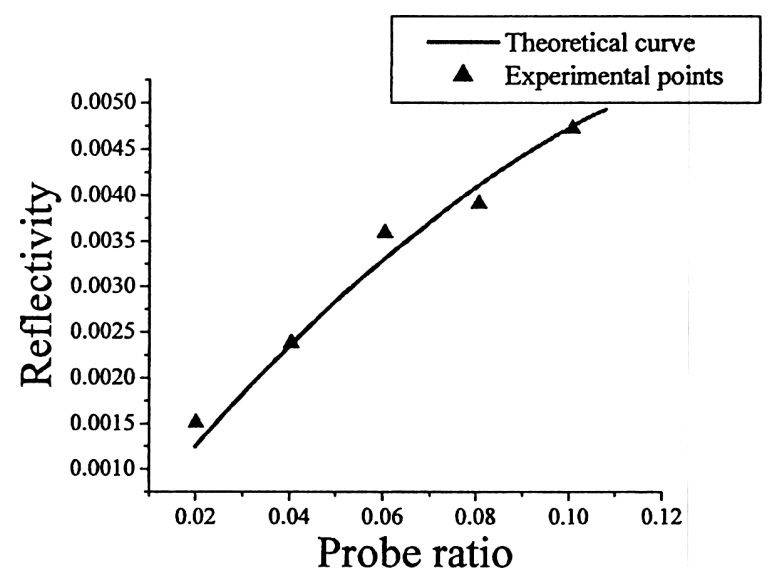

Fig. 4. Phase conjugate reflectivity vs. probe beam ratio $q$ $\left(r=1.428 \times 10^{-1}, L=10 \mathrm{~mm}, D=2 \mathrm{~mm}\right)$. dicated above are used. An agreement between the theoretical curve and experimental data is observed.

\subsection{Reflectivity vs. external electric field}

In this case, the probe beam ratio remains fixed because neither the pump beams nor the speckle beam average intensity are changed. Also the speckle size remains unmodified. The pump beam ratio is $r=1.32 \times 10^{-1}$, the probe beam ratio is $q=0.1$, the crystal thickness $L$ is $10 \mathrm{~mm}$ and a $2-\mathrm{mm}$ lens pupil aperture diameter is employed. Under these conditions, Fig. 5 shows the theoretical curve as well as the experimental values of the phase conjugate reflectivity in terms of the applied field which varies between 1 and $8 \mathrm{kV}$. To perform the theoretical calculations, the same parameters as those employed in the experiments are used. In Fig. 5, it is observed that an applied field increase produces a reflectivity increase. The experimental data almost coincide with the theoretical prediction. However, some discrepancies exist at low field values.

\subsection{Reflectivity vs. lens pupil aperture diameter}

It is clear that by changing the lens pupil aperture diameter it is possible to vary the speckle

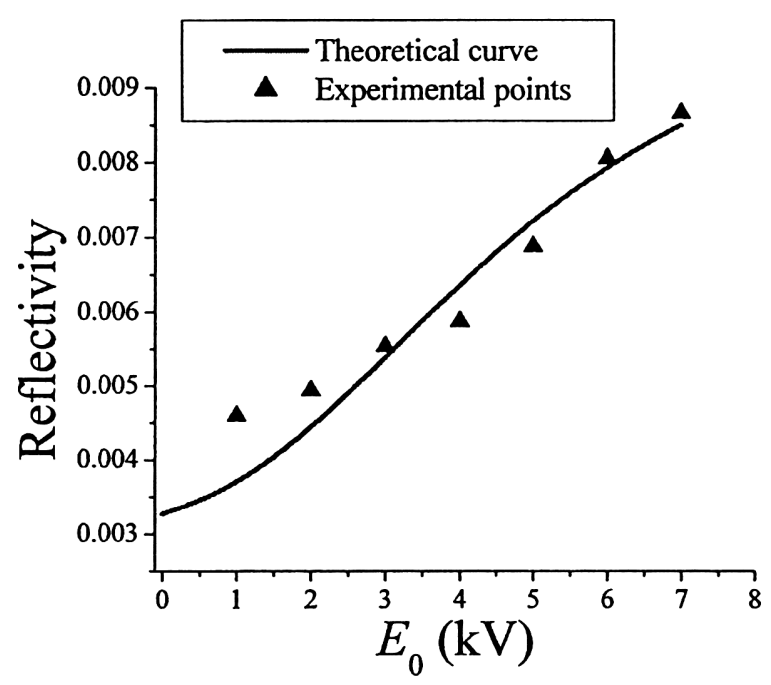

Fig. 5. Phase conjugate reflectivity vs. external applied field $\left(r \equiv 1.32 \times 10^{-1}, q=0.1, L=10 \mathrm{~mm}, D=2 \mathrm{~mm}\right)$. 
grain size, in particular the speckle depth and thereby the index grating length that modulates each registered speckle grain. Besides, when the pupil aperture diameter increases, the speckle beam average intensity increases too. So that the probe beam ratio changes. Also, the volume of the speckle grains diminishes and the speckle grain grating length reduces itself, whereas the number of speckle grains increases.

In Figs. 6 and 7, the phase conjugate reflectivity vs. the lens pupil aperture diameter is displayed by setting the pump beam ratio at $r \equiv-1.32 \times 10^{-1}$. In both cases, the BSO crystal thickness $L$ is fixed at $10 \mathrm{~mm}$. In both figures, the theoretical curve and the experimental data are shown. In Fig. 6, no external electric field is applied to the crystal $\left(E_{0}=0 \mathrm{kV}\right)$, whereas in Fig. $7, E_{0}=7 \mathrm{kV}$. As it was mentioned above, if the pupil aperture diameter increases, the average speckle intensity increases and therefore, as the pump beam values remain fixed, the probe beam increases as well. In Fig. 4, it is observed that when the probe beam increases while maintaining the pupil aperture diameter fixed, the reflectivity increases. In Figs. 6 and 7 , the probe beam increases but the reflectivity decreases. Note that in these cases, the pupil aperture diameter is not fixed and the decrease is a consequence of the speckle volume diminishing. By comparing both figures, it is apparent that the application of an external applied field increases

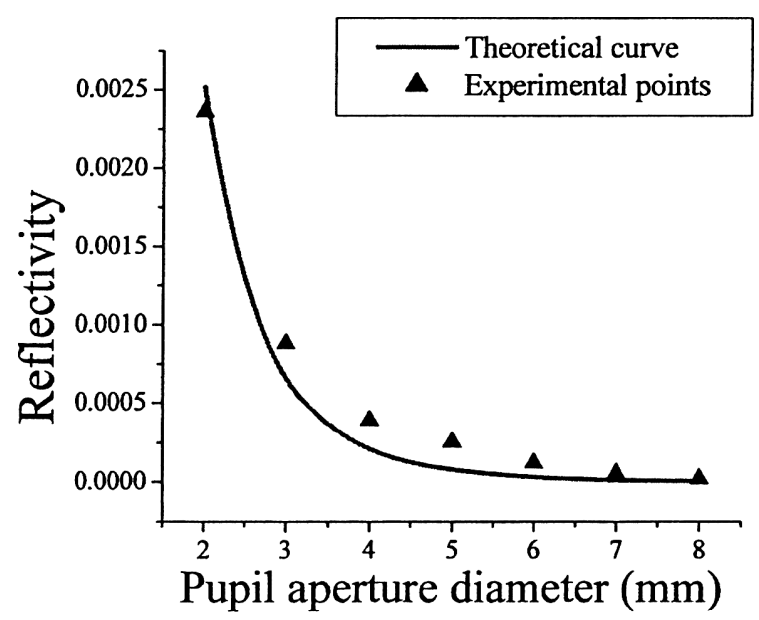

Fig. 6. Phase conjugate reflectivity vs. lens pupil aperture diameter. $\left(E_{0}=0 \mathrm{kV}, L=10 \mathrm{~mm}, r=1.32 \times 10^{-1}\right)$.

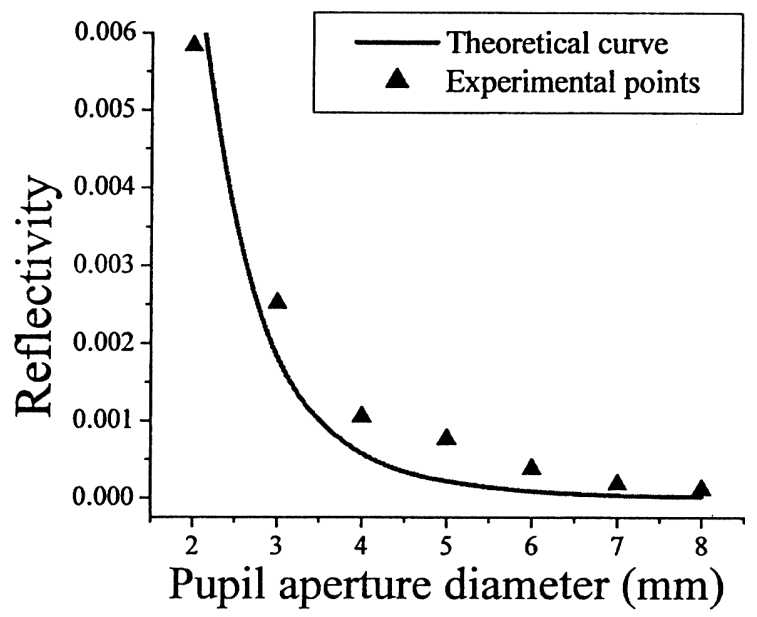

Fig. 7. Phase conjugate reflectivity vs. lens pupil aperture diameter $\left(E_{0}=7 \mathrm{kV}, L=10 \mathrm{~mm}, r=1.32 \times 10^{-1}\right)$.

the reflectivity, no matter the size of the speckle grain. It is observed that the increase in the aperture diameter and the corresponding decrease in the speckle length produces a reflectivity reduction which takes place independently on the increase in the probe beam ratio. Note that the same speckle size features described in this section are observed in the results of Fig. 3.

\section{Conclusions}

A four-wave mixing experiment produced in a BSO crystal where the signal beam bears a speckle distribution is analyzed. The speckle grains are fringe modulated due to the interference between the signal and pump beams.

The speckle pattern has a random intensity and phase distribution which leads to a local phase conjugate reflectivity. Besides, the reconstructed beam is a phase conjugate beam which is a speckle pattern itself governed by the same statistics as the input beam.

To consider this point an average amplitude and intensity must be employed in the phase conjugate reflectivity calculations.

Usually, the phase conjugate reflectivity depends on the crystal depth. In our case, the gratings are generated inside the speckle grains. This feature implies that in the theoretical calculation, if 
the speckle length is shorter than the crystal depth, then the crystal depth must be replaced by the speckle length in the reflectivity expression.

A standard theoretical model which includes these features describes adequately the phase conjugate reflectivity behavior in terms of different parameters like probe beam ratio, external applied field, and speckle size. The linear model utilized predicts correctly the experimental average values.

The precise knowledge of the reflectivity behavior leads to the adequate selection of the writein parameters in speckle applications concerning optical signal processing and metrology. An interesting application is related to the optical encryption techniques [9] that can play an important role in holographic memory systems in providing information security. As it is well known an uncorrelated set of reference beams generated by speckle patterns can be used to make it difficult to reconstruct the original beam if the codes are unknown. A possible recovering of the encrypted information could be done by a phase conjugate read out scheme. In this sense, the analysis of a phase conjugate speckle arrangement is a preliminary step to the potential use of this approach in optical encryption arrangement.

\section{Acknowledgements}

This research was performed under the auspices of CONICET, Fundación Antorchas and Facultad Ingeniería, Universidad Nacional de La Plata (Argentina). A. Salazar acknowledges the financial support of the Universidad Pontificia Bolivariana of Medellín, Colombia and gratefully acknowledges Prof. Rafael Cabanzo and Prof. Alberto Lencina for many technical discussions.

\section{References}

[1] P. Yeh, Introduction to Photorefractive Nonlinear Optics, Wiley, New York, 1993.

[2] P. Huignard, P. Günter, in: P. Huignard, P. Günter (Eds.), Photorefractive Material and their Applications I-II, Springer, Berlin, 1989.

[3] L. Solymar, D.J. Webb, A. Grunnet-Jepsen, The Physics and Applications of Photorefractive Materials, Clarendon Press, Oxford, 1996.

[4] J.P. Huignard, J.P. Herriau, P. Aubourg, E. Spitz, Opt. Lett. 4 (1979) 21.

[5] J.P. Huignard, J.P. Herriau, J.P. Pichon, A. Marrakchi, Opt. Lett. 5 (1980) 436.

[6] J.P. Huignard, H. Rajbenbach, P. Refregier, L. Solymar, Opt. Eng. 24 (1985) 586.

[7] J. Feinberg, Opt. Lett. 8 (1983) 569.

[8] Xiaodi Tan, Osamu Matoba, Tsutomu Shimura, Kazuo Kuroda, Bahram Javidi, Appl. Opt. 39 (2000) 6689.

[9] G. Unnikrishnan, J. Joseph, K. Singh, Appl. Opt. 37 (1998) 8181.

[10] Y.H. Ja, Opt. Laser Technol. 17 (1985) 36.

[11] M. Françon, Laser Speckle and Applications in Optics, Academic Press, New York, 1979.

[12] R.K. Erf, Speckle Metrology, Academic Press, New York, 1978.

[13] H.J. Tiziani, K. Leonhardt, J. Klenk, Opt. Commun. 34 (1980) 327.

[14] H.J. Tiziani, J. Klenk, Appl. Opt. 20 (1981) 1467.

[15] R. Tripathi, G.S. Pati, A. Kumar, K. Singh, Opt. Commun. 149 (1998).

[16] H.J. Tiziani, Opt. Acta 29 (1982) 463.

[17] M. Tebaldi, L. Angel Toro, M.C. Lasprilla, N. Bolognini, Opt. Commun. 155 (1998) 342.

[18] L. Angel, M. Tebaldi, M. Trivi, N. Bolognini, Opt. Commun. 168 (1999) 55.

[19] M. Tebaldi, L. Angel, M. Trivi, N. Bolognini, Opt. Eng. 39 (2000) 3232.

[20] M. Cronin-Golomb, B. Fischer, J.O. White, A. Yariv, IEEE J. Quantum Electron. QE-20 (No. 1) (1984) 241.

[21] N.V. Kukhtarev, V.B. Markov, S.G. Odulov, M.S. Soskin, V.L. Vinetskii, Ferroelectrics 22 (1979) 949.

[22] J.P. Huignard, J.P. Herriau, G. Rivet, P. Günter, Opt. Lett. 5 (1980) 102. 